\title{
Prognostic Significance of Vascular Endothelial Growth Factor-A (VEGF-A) and Ki-67 Expression in Head and Neck Cancer Patient with Negative Neck
}

\author{
Vipa Boonkitticharoen1, Boonchu Kulapaditharom², Noppadol Larbcharoensub ${ }^{3}$, \\ Phurich Praneetvatakul ${ }^{2}$, Thongchai Bhongmakapat ${ }^{2}$ \\ ${ }^{1}$ Department of Diagnostic and Therapeutic Radiology, Ramathibodi Hospital, Mahidol University, \\ Bangkok, Thailand \\ ${ }^{2}$ Department of Otolaryngology, Ramathibodi Hospital, Mahidol University, Bangkok, Thailand \\ ${ }^{3}$ Department of Pathology, Ramathibodi Hospital, Mahidol University, Bangkok, Thailand \\ Email: vipa.bon@mahidol.ac.th
}

Received 10 August 2015; accepted 20 September 2015; published 23 September 2015

Copyright (C) 2015 by authors and Scientific Research Publishing Inc.

This work is licensed under the Creative Commons Attribution International License (CC BY). http://creativecommons.org/licenses/by/4.0/

(c) (i) Open Access

\section{Abstract}

Background: Management of NO neck in patients with head and neck squamous cell carcinoma (HNSCC) remains a subject of continued debate. Prognostic biomarkers might provide useful information for treatment selection and adjustment. Objective: To evaluate the prognostic relevance of VEGF-A and Ki-67 expression to types of neck management. Methods: This prospective study included 140 patients with HNSCC. Tumor expression of VEGF-A and Ki-67 was measured by immunohistochemistry. Based on tumor size and site criteria, 88 patients with NO neck were categorized as high, intermediate and low risk of subclinical neck diseases and accordingly treated by elective neck dissection (END), irradiation (ENI) and observation. Adjuvant treatment was given to tumor with close or positive margins. A multivariate Cox regression model was used to identify prognostic factors. Impact of biomarker expression, treatment type and risk category on disease-specific survival (DSS) in the setting of NO neck were evaluated by Kaplan-Meier survival and adjusted hazard ratio (HR). Results: Coexpression of VEGF-A and Ki-67 (HR $=2.351, p=0.021$ ) and positive node (HR $=2.301, p=0.009)$ were independent prognostic factors for HNSCC. In the setting of NO neck, marker coexpression has an HR of $4.97(p=0.004)$ independent of treatment modalities $(p=0.069)$ and risk categories $(p=0.971)$. Alternatively, neither marker expression was predictive of a better treatment outcome for END compared to ENI, as suggested by the odds of patients being survived 15.4 times greater $(p=0.01)$ and the 5 -year DSS rates of $85.1 \%$ versus 44.7\% ( $p=0.008)$. Conclusion: Coexpression of VEGF-A and Ki-67 is a suggestion of tumor microinvasiveness in addition to risk of lymph node metastasis and may indicate the need of adjuvant

How to cite this paper: Boonkitticharoen, V., Kulapaditharom, B., Larbcharoensub, N., Praneetvatakul, P. and Bhongmakapat, T. (2015) Prognostic Significance of Vascular Endothelial Growth Factor-A (VEGF-A) and Ki-67 Expression in Head and Neck Cancer Patient with Negative Neck. International Journal of Otolaryngology and Head \& Neck Surgery, 4, 356-368. http://dx.doi.org/10.4236/ijohns.2015.45060 
treatment despite negative tumor margins. Neither marker expression serves an indicator for the selection of END over ENI in neck management.

\author{
Keywords \\ Head and Neck Squamous Cell Carcinoma, Negative Neck Node, Neck Management, Prognostic \\ Biomarkers, VEGF-A and Ki-67
}

\title{
1. Introduction
}

Head and neck squamous cell carcinoma (HNSCC) has a high propensity to invade lymph nodes in the cervical region before the development of distant metastasis [1]. The presence of nodal metastases is the most important factor for prognosis and treatment plan [2]. In patient with clinically N0 neck (cN0), there is still a high rate of occult nodal metastasis (12\% - 50\%) which is strongly dependent of site and T class of the primary tumor [3]. For instance, T1 cancer of the oral cavity excluding the oral tongue has the lowest rate of subclinical neck disease $(<20 \%)$ as opposed to tumors of the pyriform sinus, base of tongue, supraglottic larynxes have the highest rate ( $>30 \%)$ despite their early T class [4] [5]. Based on a decision analysis taking into account the incidence of occult metastasis, disease control rate and treatment complications, treatment of the neck is advised for patient with a risk of subclinical neck disease $\geq 20 \%$ [6].

In management of patients with cN0 neck, there are three policies advocated: a watchful waiting policy for those with a risk $<20 \%$; elective neck dissection (END) or elective neck irradiation (ENI) for a risk $\geq 20 \%$; and neck treatment plus adjuvant therapy for the high risk patients [7] [8]. More than two decades since the adoption of Weiss's recommendation to treat the $\mathrm{cN} 0$ neck with a risk $\geq 20 \%$, head and neck oncologists continue to debate on the appropriate strategy in neck management. Central to the debate is the issue surrounding whether a patient with cN0 neck should be treated now or be closely observed, whether the patient should be treated by END or receive ENI [9].

Pretreatment risk stratification of patients may provide information that helps selecting the appropriate modality in neck management for a particular risk category [9]. Mendenhall et al. [4] [5] defined risk of subclinical neck diseases on the basis of tumor $\mathrm{T}$ class and anatomical location to allow the stratification of patients into three risk groups: high risk $>30 \%$; intermediate risk $20 \%-30 \%$ and low risk $<20 \%$. With an additional inclusion of prognostic markers relevant to tumor progression and metastasis, risk stratification might place the oncologist in a better position to select the most appropriate therapeutic approach for individual patients.

Recent advances in molecular biology have revealed that primary tumor is predisposed to metastasize [10]. Maintenance of primary tumor gene expression profiles in lymph node (LN) metastases [10] suggests the use of biomarkers expressing on primary tumor specimen as markers for the presence of occult LN metastases [11]. Metastasis is a highly complicated process resulting from epigenetic and genetic alterations so as to provide tumor cells with proliferative advantage, capability to evade apoptosis and to escape immune surveillance. To metastasize, tumor cells must further acquire the ability to degrade interstitial matrix, lose cell-to-cell contact, invade blood and lymph vessels and to induce angiogenesis and lymphangiogenesis [12] [13]. Proteins that have been identified as promoters of LN metastasis in HNSCC can be grouped according to their biological functions: cell cycle regulation and proliferation [e.g. epidermal growth factor receptor (EGFR), cyclin D1, Ki-67]; tumor suppressor and apoptosis [e.g. p53, p21, Bcl-2]; cell adhesion and matrix degradation [e.g. cadherins, CD44, matrix metalloproteinases (MMPs)]; tumor hypoxia [e.g. hypoxia inducible factor-1 (HIF-1), carbonic anhydrase IX (CA IX), glucose transporters (GLUT)]; angiogenesis and lymphangiogesis [e.g. VEGF-A, VEGF-C, LYE-1] [14]-[16]. Since tumor proliferation and angiogenesis are known to play important part in tumor progression and metastasis [12], VEGF-A, an angiogenesis regulator, and Ki-67, a proliferation marker, are markers of interest for their associations with LN metastasis [17]-[22] and prognosis in HNSCC [19] [21] [23]-[25]. In our previous study, increased expression of Ki-67 was associated with positive neck nodes, either expression of VEGF-A or Ki-67 was recognized in tumor with advanced T class. Coexpression of VEGF-A and Ki-67 represented an aggressive tumor phenotype for being associated to LN metastasis especially in early tumor of the oral cavity, oropharynx and hypopharynx [20].

This investigation is the continuum of a prospective study on the use of VEGF-A and Ki-67 in prediction of 
LN metastasis in HNSCC [20]. The purposes of this study were two-folds, namely: 1) to assess whether combined VEGF-A and Ki-67 expression represented an independent prognostic index for this series of surgery treated HNSCC; 2) to evaluate the impact of VEGF-A and Ki-67 expression on survival of patients with N0 neck underwent various modalities of neck management from observation, ENI, END to END plus adjuvant treatment.

\section{Patients and Methods}

\subsection{Study Overview}

This prospective study cohort has been previously described [20]. A total of 147 patients diagnosed with squamous cell carcinoma (SCC) of the head and neck were consecutively included during 2001-2005 at Department of Otolaryngology, Ramathibodi Hospital. The inclusion criteria were treatment-naive patients with resectable SCC of the oral cavity, oropharynx, hypopharynx and larynx, stage I-IV. Patients with distant metastasis or recurrence disease were excluded. Pretreatment tumor specimens were obtained upon the agreement with Ramathibodi Hospital Ethics Committee in the use of tumor specimens for scientific analysis and receiving patients' written informed consents. The paraffin-embedded tumor specimens were stained for the expression of VEGF-A and Ki-67 by immunohistochemistry. The techniques of tissue staining and methods for determination of marker expression have been described in details in our previous study [20].

To evaluate whether VEGF-A and Ki-67 expression could serve as a significant prognostic indicator for the present series of patients with surgery treated tumor of the head and neck, demographic (age and gender), and clinicopathological factors (tumor grade and location, $\mathrm{T}$ stage and nodal status) were included in survival analysis. Of a total of 147 patients included previously, only 140 were eligible for study because of the availability of complete data for survival analysis. Of these 140 patients, 52 (37.1\%) had pathologically confirmed positive neck nodes and 88 (62.9\%) had negative nodes either clinically defined or pathologically confirmed. Demographic, clinicopathological characteristics and biomarker expression in patients with positive and negative necks are presented in Table 1.

Table 1. Demographic, clinicopathologic and biological variables in association with nodal status.

\begin{tabular}{|c|c|c|c|c|}
\hline Variable & Total, $\mathrm{n}$ & Node negative n (\%) & Node positive n (\%) & $p$ \\
\hline \multicolumn{5}{|l|}{ Age at diagnosis } \\
\hline$\leq 64$ years & 73 & 43 (58.9) & $30(41.1)$ & \multirow[t]{2}{*}{0.40} \\
\hline$>64$ years & 67 & $45(67.2)$ & $22(32.8)$ & \\
\hline \multicolumn{5}{|l|}{ Sex } \\
\hline Male & 112 & $73(34.8)$ & $39(65.2)$ & \multirow[t]{2}{*}{0.36} \\
\hline Female & 28 & $15(46.4)$ & 13 (53.6) & \\
\hline \multicolumn{5}{|l|}{ Tumor site } \\
\hline Oral cavity & 57 & $40(70.2)$ & $17(29.8)$ & \multirow{3}{*}{$<<0.0001$} \\
\hline Pharynx & 27 & $3(11.1)$ & $24(88.9)$ & \\
\hline Larynx & 56 & $45(80.4)$ & $11(19.6)$ & \\
\hline \multicolumn{5}{|l|}{ Differentiation } \\
\hline Well & 89 & 64 (71.9) & $25(28.1)$ & \multirow[t]{2}{*}{0.014} \\
\hline Moderate and poor & 51 & $24(47.1)$ & 27 (52.9) & \\
\hline \multicolumn{5}{|l|}{ T stage } \\
\hline $\mathrm{T} 1,2$ & 62 & $46(74.2)$ & $16(25.8)$ & \multirow[t]{2}{*}{0.021} \\
\hline $\mathrm{T} 3,4$ & 78 & $42(53.8)$ & $36(46.2)$ & \\
\hline \multicolumn{5}{|l|}{ VEGF-A } \\
\hline High $>2.74$ & 55 & $25(45.5)$ & $30(54.5)$ & \multirow[t]{2}{*}{0.001} \\
\hline Low $\leq 2.74$ & 85 & $63(74.1)$ & $22(25.9)$ & \\
\hline \multicolumn{5}{|l|}{ Ki-67 } \\
\hline High $>58.61 \%$ & 55 & $31(56.4)$ & $24(43.6)$ & \multirow[t]{2}{*}{0.21} \\
\hline Low $\leq 58.61 \%$ & 85 & $58(68.2)$ & 27 (31.8) & \\
\hline \multicolumn{5}{|l|}{ Combined expression } \\
\hline Both high & 27 & $11(40.7)$ & $16(59.3)$ & \multirow{3}{*}{0.008} \\
\hline Either high & 59 & $36(61)$ & $23(39)$ & \\
\hline Neither high & 54 & $41(75.9)$ & $13(24.1)$ & \\
\hline
\end{tabular}




\subsection{Risk Category and Treatment of Patients with NO Neck}

Eighty-eight patients with N0 neck were grouped as high risk, intermediate risk and low risk of occult neck diseases according to risk definition defined by Mendenhall et al. [4] [5]. The high risk group (>30\%) includes T1-4 pyriform sinus and base of tongue; T2-4 oropharynx (soft palate and tonsil), hypopharynx (pharyngeal wall) and supraglottic larynx; T3-4 oral cavity (floor of mouth, tongue, retro molar trigone, gingival, hard palate and buccal mucosa); T4 glottis. The intermediate risk group (20\% - 30\%) comprises T1-2 oral tongue; T1 oropharynx, hypopharynx and supraglottic larynx; T2 oral cavity and T3 glottis. The low risk group ( $<20 \%)$ encompasses T1 oral cavity and T1-2 glottis.

Table 2 shows the risk groups in this series.

Elective treatment of the neck was performed in patient with a risk of subclinical neck disease $\geq 20 \%$ [6]. Initially, elective neck dissection (END) was conducted for patients with cN0 neck (105 cases) at the time of primary surgery. Post-operative radiotherapy (PORT) was given to patients of the high risk group with the following features: tumor with closed surgical margins, T3-4 tumor of the oropharynx, hypopharynx and supraglottic larynx. Concomitant chemoradiotherapy (CCRT) using cisplatin was administered to patient with positive tumor margins. Patients of the intermediate risk group including T3-4 tumor with negative margins were either treated by END or elective neck irradiation (ENI). No neck treatment was given to the low risk group except for those T1 cancers of the floor of mouth and T2 glottic cancer to which ENI was prescribed. Patients (17 cases) with metastatic nodal diseases uncovered subsequently by histologic examination of the dissected lymph node specimens were excluded from this study (Table 3).

Table 2. Risk category according to tumor T class and location [4] [5].

\begin{tabular}{ccccc}
\hline Risk category & Occult neck diseases & $\mathrm{T}$ & Primary tumor site & $\mathrm{n}(\%)$ \\
\hline High & $>30 \%$ & $\mathrm{~T} 3-4$ & Pyriform sinus and base of tongue & $3(8.3 \%)$ \\
$\mathrm{n}=36$ & & $\mathrm{~T} 3-4$ & Lip, buccal mucosa, tongue and hard palate & $11(30.6 \%)$ \\
& & $\mathrm{T} 2-4$ & Supraglottis & $10(27.8 \%)$ \\
& & $\mathrm{T} 4$ & Glottis & $11(30.5 \%)$ \\
Intermediate & $20 \%-30 \%$ & $\mathrm{~T} 3$ & Subglottis & $1(2.8 \%)$ \\
$\mathrm{n}=29$ & & $\mathrm{~T} 2$ & Lip, buccal mucosa and hard palate & $3(10.3 \%)$ \\
& & T1-2 & Tongue & $18(62.1 \%)$ \\
Low & & Glottis & $8(27.6 \%)$ \\
$\mathrm{n}=23$ & $\mathrm{~T} 1$ & Lip and floor of mouth & $8(34.78 \%)$ \\
& & $\mathrm{T} 1-2$ & Glottis & $14(60.87 \%)$ \\
\end{tabular}

Table 3. Biomarker expression profiles and types of neck management in patients with different risks of subclinical neck diseases. Data show number (percent).

\begin{tabular}{|c|c|c|c|c|}
\hline & High risk & Intermediate risk & Low risk & \multirow{2}{*}{$p$} \\
\hline & $\mathrm{n}=36$ & $n=29$ & $\mathrm{n}=23$ & \\
\hline VEGF-A and Ki-67 & & & & 0.002 \\
\hline Both high & $2(5.6)$ & $5(17.2)$ & $4(17.4)$ & \\
\hline Either high & 23 (63.9) & $5(17.2)$ & $8(34.8)$ & \\
\hline Neither high & $11(30.6)$ & $19(65.5)$ & $11(47.8)$ & \\
\hline \multicolumn{5}{|l|}{ Neck management } \\
\hline END + Adj Tx & $17(47.2)$ & - & - & 0.000 \\
\hline ENI & $11(30.6)$ & $15(51.7)$ & $7(30.4)$ & \\
\hline END & $8(22.2)$ & $14(48.3)$ & - & \\
\hline Observation & - & - & $16(69.6)$ & \\
\hline
\end{tabular}

END + Adj Tx: Elective neck dissection + adjuvant treatment; ENI: Elective neck irradiation; END: Elective neck dissection. 


\subsection{Follow-Up and Time-to-Event Measurement}

The follow-up started after treatment completion in every 2 months during the first year, every 3 months in the second and every 6 months thereafter. Disease-specific survival (DSS) was the endpoint of outcome for this study. Time-to-event was defined as the duration since the date of diagnosis to the date of tumor-related death or the date of last follow-up for patients who were still alive. Data were censored for patients who were alive at the end of study; lost to follow-up; dead from causes not attributable to cancer. This study had a median follow-up time of 44 months (range, 1 - 102.1 months).

\subsection{Biomarker Cutoffs for Prognostic Study}

The expression of Ki-67 was determined by the percentage of positive tumor cells in hot spot area. For VEGF-A, a score varying from 0 to 4 was graded on the basis of stained intensity and percentage of cell stained [20]. In general, a median value is arbitrarily chosen as a cutoff for biomarker expression [19] [20]. In this prognostic study, optimal cutoff was chosen at a level yielding the most significant hazard ratio (HR) adjusted for potential confounding factors including age at diagnosis, gender, tumor site, tumor differentiation, T stage and nodal status. To this end, cutoffs for biomarker expression were step-wisely increased from the $50^{\text {th }}$ percentile up to the $70^{\text {th }}$ percentile with a five percentile increment. In the context of combined marker expression, the most significant HR of $2.357(p=0.021)$ was achieved at the $60^{\text {th }}$ percentile cutoff, i.e. VEGF-A score of 2.74 and Ki-67 labeling index of $58.61 \%$.

\subsection{Statistical Analysis}

Comparison of categorical data between groups was conducted by $\chi^{2}$ or Fisher Exact test as appropriate. Survival analyses were performed using Predictive Analytics Software (PASW) statistics 18 (SPCC Inc., Chicago, IL, USA). To identify independent prognostic factors for this patient cohort, univariate and multivariate analyses were carried out using Cox proportional hazard regression model. Besides biomarker expression, the survival analysis also included factors generally known to influence survival of head and neck cancer patients. These factors are age at diagnosis, sex, tumor site, tumor differentiation, T stage and nodal status [26]. Significant prognostic factors were judged from the value of HR $>1$ and the level of significance at $p<0.05$. The final prognostic model was constructed by including variables with $p$ values $\leq 0.1$.

In the setting of N0 neck, impact of risk category, type of neck management and biomarker expression on DSS was assessed by Kaplan-Meier survival curve, and multivariate Cox regression model. All statistical tests were two-tailed and $p$ value $<0.05$ was considered significant.

\section{Results}

\subsection{Prognostic Factors for HNSCC}

The median follow-up time for this study was 44 months (range, 1 - 102.1 months). During follow-up periods, a total of 70 patients died: 60 due to HNSCC, 10 from tumor-unrelated causes such as diseases of the lung and liver, heart attack, old age, etc; 16 patients were lost to follow-up; 44 patients were alive at the time of last contact. The impact on disease-specific survival (DSS) was evaluated for a number of potential influencing factors including age at diagnosis, sex, primary tumor site, tumor differentiation, $\mathrm{T}$ stage, nodal status, and biomarker expression using univariate and multivariate Cox proportion hazard regression models. Results of the analyses are presented in Table 4. In univariate analysis, significant associations with poor DSS were observed for tumor of the oral cavity and pharynx in contrasting to the larynx ( $\mathrm{HR}=1.836, p=0.029)$, positive lymph node $(\mathrm{HR}=$ 3.022, $p=0.000$ ), increase expression of VEGF-A ( HR $=1.932, p=0.001)$, Ki-67 (HR $=1.744, p=0.032)$ and coexpression of VEGF-A and Ki-67 (HR $=2.987, p=0.001$ ). In multivariate analysis, only positive lymph node $(\mathrm{HR}=2.301, p=0.009)$ and biomarker coexpression $(\mathrm{HR}=2.357, p=0.021)$ remained significant.

\subsection{Impact of Risk Category, Biomarker Expression and Modality of Neck Management on Survival of Patients with NO Neck}

In univariate survival analysis, combined marker expression $(p=0.001)$ and modality of neck management $(p=$ $0.014)$ were significantly affecting DSS in contrasting to the nonsignificant effect of the risk category $(p=0.618)$ (Figures 1-3). Multivariate Cox regression analysis revealed an overall significant $p$ level of 0.013 for VEGF-A 


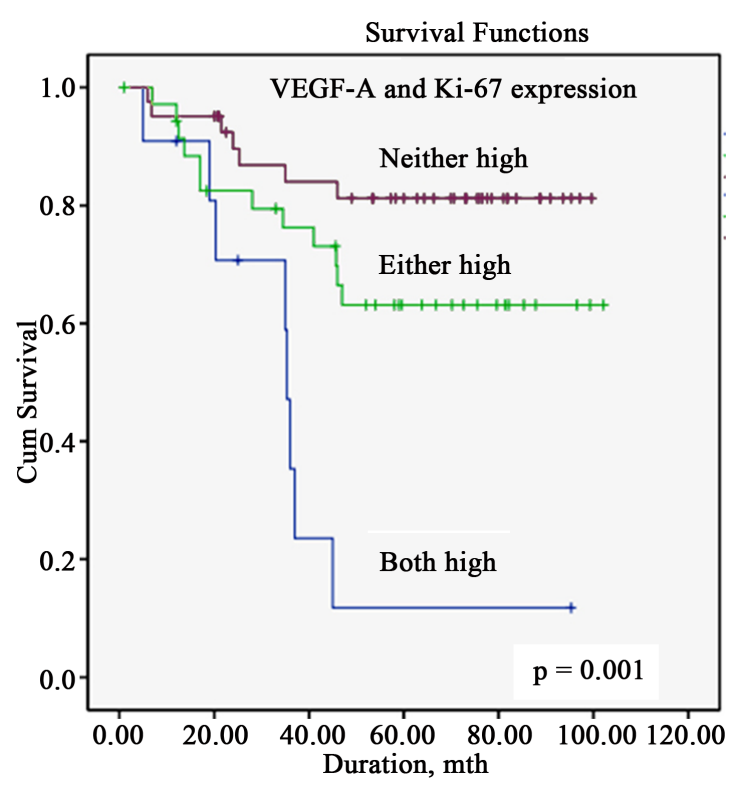

Figure 1. Kaplan-Meier survival curves for patients with tumor expressing both VEGF-A and Ki-67, either and neither markers.

Table 4. Cox proportional hazard regression analyses of prognostic factors for disease-specific survival (DSS).

\begin{tabular}{|c|c|c|c|c|}
\hline \multirow{2}{*}{ Variable } & \multicolumn{2}{|c|}{ Univariate } & \multicolumn{2}{|c|}{ Multivariate Multivariate } \\
\hline & HR (95\% CI) & $p$ & HR (95\% CI) & $p$ \\
\hline \multicolumn{5}{|l|}{ Age at diagnosis } \\
\hline$>64$ years & $1.086(0.654-1.801)$ & 0.751 & $1.289(0.765-2.173)$ & 0.340 \\
\hline$\leq 64$ years & 1 & & 1 & \\
\hline \multicolumn{5}{|l|}{ Sex } \\
\hline Male & $0.848(0.458-1.568)$ & 0.599 & $1.253(0.653-2.402)$ & 0.498 \\
\hline Female & 1 & & 1 & \\
\hline \multicolumn{5}{|l|}{ Tumor site } \\
\hline Oral and pharynx & $1.836(1.063-3.169)$ & 0.029 & $1.650(0.880-3.092)$ & 0.118 \\
\hline Larynx & 1 & & 1 & \\
\hline \multicolumn{5}{|l|}{ Differentiation } \\
\hline Moderate and poor & $1.11(0.66-1.869)$ & 0.694 & $0.705(0.401-1.238)$ & 0.223 \\
\hline Well & 1 & & 1 & \\
\hline \multicolumn{5}{|l|}{ T stage } \\
\hline T3,4 & $1.43(0.85-2.408)$ & 0.178 & $1.239(0.701-2.189)$ & 0.461 \\
\hline $\mathrm{T} 1,2$ & 1 & & 1 & \\
\hline \multicolumn{5}{|l|}{ Nodal status } \\
\hline $\mathrm{N}+$ & $3.022(1.809-5.049)$ & 0.000 & $2.301(1.231-4.304)$ & 0.009 \\
\hline N0 & 1 & & 1 & \\
\hline \multicolumn{5}{|l|}{ VEGF-A } \\
\hline High $>2.74$ & $1.932(1.161-3.215)$ & 0.011 & $1.435(0.807-2.553)$ & 0.219 \\
\hline Low $\leq 2.74$ & 1 & & 1 & \\
\hline \multicolumn{5}{|l|}{ Ki-67 } \\
\hline High > 58.61 & $1.744(1.05-2.898)$ & 0.032 & $1.559(0.929-2.615)$ & 0.093 \\
\hline Low $\leq 58.61 \%$ & 1 & & 1 & \\
\hline \multicolumn{5}{|l|}{ Combined expression } \\
\hline Both high & $2.987(1.569-5.687)$ & 0.001 & $2.357(1.14-4.873)$ & 0.021 \\
\hline Either high & $1.241(0.665-2.314)$ & 0.498 & $1.187(0.559-2.353)$ & 0.624 \\
\hline Neither high & 1 & & 1 & \\
\hline
\end{tabular}

HR: Hazard ratio; CI: Confidence interval. 


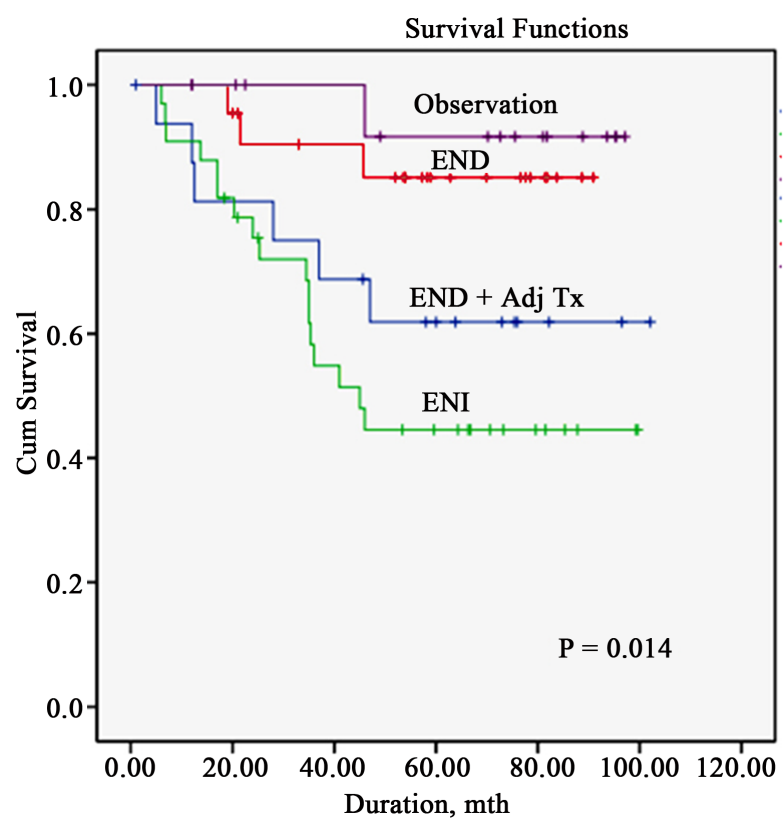

Figure 2. Kaplan-Meier survival curves for patients underwent different neck managements from elective neck dissection plus adjuvant treatment (END + Adj Tx), elective neck dissection (END), elective neck irradiation (ENI) to observation.

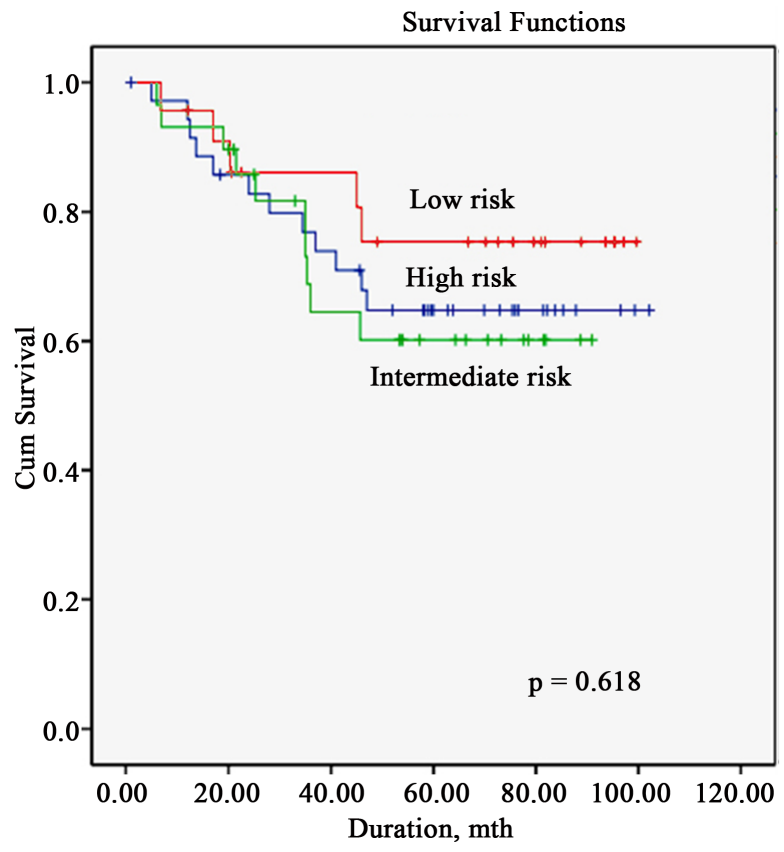

Figure 3. Kaplan-Meier survival curves for patients categorized as low, intermediate and high risks of subclinical neck diseases.

and Ki-67 expression. In relative to tumor with neither marker expression, only coexpression of VEGF-A and Ki-67 was observed with a significant HR of $4.97(p=0.004)$. For the modality of neck management, the impact was at a marginally significant level with a p value of 0.069 . In comparing different types of neck treatment to observation, significant HR of $8.737(p=0.036)$ was obtained for neck treatment by ENI, and a marginally significant HR of $6.043(p=0.098)$ for END + adjuvant treatment (Table 5). 
Table 5. Multivariate Cox regression analysis of the impact of biomarker expression and neck management on disease-specific survival.

\begin{tabular}{|c|c|c|c|c|}
\hline \multirow{2}{*}{ Variable } & \multirow{2}{*}{ HR } & \multicolumn{2}{|c|}{$95 \%$ CI } & \multirow{2}{*}{$p$} \\
\hline & & Lower & Upper & \\
\hline VEGF-A and Ki-67 & & & & 0.013 \\
\hline Both high & 4.97 & 1.669 & 14.804 & 0.004 \\
\hline Either high & 1.659 & 0.651 & 4.41 & 0.28 \\
\hline Neither high & 1 & - & - & - \\
\hline Neck management & & & & 0.069 \\
\hline END + Adj Tx & 6.043 & 0.717 & 50.911 & 0.098 \\
\hline ENI & 8.737 & 1.152 & 66.263 & 0.036 \\
\hline END & 2.621 & 0.269 & 25.504 & 0.407 \\
\hline Observation & 1 & - & - & - \\
\hline
\end{tabular}

HR: Hazard ratio; CI: Confidence interval; END + Adj Tx: Elective neck dissection + adjuvant treatment; ENI: Elective neck irradiation; END: Elective neck dissection.

\subsection{Dependence of Treatment Outcomes on Biomarker Expression and Type of Neck Management}

Table 6 shows how different types of neck management and patterns of combined marker expression influencing the treatment outcomes in patients with different risks of occult neck diseases. Mutual dependence among treatment outcomes, types of neck treatment and patterns of combined marker expression were tested using a $2 \times$ $2 \times 3$ three-dimensional contingency table. By pair-wise comparison, results of $\chi^{2}$ analyses of contingency tables are presented in Table 7 along with log-rank test of the Kaplan-Meier survival curves.

There were two important observations according to data shown in Table 6. Patients with coexpression of VEGF-A and Ki-67 had a great chance $(8 / 11=72.7 \%)$ to die regardless the type of neck management. On the other hand, tumors expressing neither marker were better controlled by END (with and without adjuvant treatment) than those treated by ENI. The odds of patients being alive/censored in END group were 15.4 times greater than the ENI group $(p=0.01)$. This offered an explanation why the survival for patients in the END treated group was superior to the ENI group (Figure 2). Five-year DSS rates for END and ENI given to patients with similar risks of occult neck diseases, i.e. intermediate to high risks, were $85.1 \%$ and $44.7 \%$, respectively (p $=0.008$ ). Radio-biologically, dividing cells are more radiosensitive than the nondividing cells. Therefore, ENI was less effective in eradicating the nondividing metastatic cells disseminated from tumor expressing neither markers. This was in contrasting to END which involved surgical removal of lymph nodes with occult metastases regardless their radiobiological property. Equivalent treatment outcomes between END and observation would implicate the effectiveness of END in removal of the subclinical neck diseases to achieve an outcome comparable to that of the observation group with low risk of occult neck diseases. This was further supported by the treatment outcome in END + adjuvant treatment group. Despite the unfavorable biomarker expression profiles similar to those of the ENI group $(p=0.165)$ and the clinicopathologically severe features in this group (i.e. high risk anatomical location and close or positive tumor margins), effective removal of subclinical neck diseases by END particularly in patients expressing neither markers helped improving patient survival. Pair-wise comparison of Kaplan-Meier survival curves between END + adjuvant treatment versus END or observation were nonsignificant, with $p$ values of 0.099 and 0.052 , respectively. Five-year DSS rates among these groups were $61.9 \%$ for END + adjuvant treatment, 85.1\% for END and 91.7\% for observation.

\section{Discussion}

Management of the negative neck in patients with HNSCC is a subject of extensive debate. Issue central to the debate usually involves the question whether END would offer a survival advantage over the policy of watchful waiting. When there is an indication for neck treatment, should a patient be treated with END or ENI [19]. 
Table 6. Biomarker expression in association with treatment outcomes in patients of different risk categories underwent various types of neck management. Data show number of patients.

\begin{tabular}{|c|c|c|c|c|c|c|c|c|c|c|}
\hline \multirow{2}{*}{ Neck management } & \multirow{2}{*}{$\begin{array}{c}\text { VEGF-A } \\
\text { and } \\
\text { Ki-67 }\end{array}$} & \multicolumn{3}{|c|}{ High risk } & \multicolumn{3}{|c|}{ Intermediate risk } & \multicolumn{3}{|c|}{ Low risk } \\
\hline & & Dead & Censored & Total & Dead & Censored & Total & Dead & Censored & Total \\
\hline \multirow{4}{*}{ END + Adj Tx } & Both & 2 & 0 & 2 & - & - & - & - & - & - \\
\hline & Either & 4 & 7 & 11 & - & - & - & - & - & - \\
\hline & Neither & 0 & 4 & 4 & - & - & - & - & - & - \\
\hline & Total & 6 & 11 & 17 & - & - & - & - & - & - \\
\hline \multirow[t]{4}{*}{ ENI } & Both & 0 & 0 & 0 & 3 & 1 & 4 & 2 & 0 & 2 \\
\hline & Either & 4 & 5 & 9 & 1 & 1 & 2 & 1 & 2 & 3 \\
\hline & Neither & 2 & 0 & 2 & 3 & 6 & 9 & 1 & 1 & 2 \\
\hline & Total & 6 & 5 & 11 & 7 & 8 & 15 & 4 & 3 & 7 \\
\hline \multirow[t]{4}{*}{ END } & Both & 0 & 0 & 0 & 1 & 0 & 1 & - & - & - \\
\hline & Either & 0 & 3 & 3 & 1 & 2 & 3 & - & - & - \\
\hline & Neither & 0 & 5 & 5 & 1 & 9 & 10 & - & - & - \\
\hline & Total & 0 & 8 & 8 & 3 & 11 & 14 & - & - & - \\
\hline \multirow[t]{4}{*}{ Observation } & Both & - & - & - & - & - & - & 0 & 2 & 2 \\
\hline & Either & - & - & - & - & - & - & 1 & 4 & 5 \\
\hline & Neither & - & - & - & - & - & - & 0 & 9 & 9 \\
\hline & Total & - & - & - & - & - & - & 1 & 15 & 16 \\
\hline
\end{tabular}

END + Adj Tx: Elective neck dissection + adjuvant treatment; ENI: Elective neck irradiation; END: Elective neck dissection.

Table 7. Pair-wise comparison between treatment outcomes in terms of Kaplan-Meier survival or frequency data presented in a $2 \times 2 \times 3$ three-dimensional contingency table. Data show $p$ values of the tests.

\begin{tabular}{cccccc}
\hline & Analysis & END + Adj Tx & ENI & END & Observation \\
\hline END + Adj Tx & Kaplan-Meier survival & - & 0.308 & 0.099 & 0.052 \\
& Contingency table & - & 0.165 & $<0.001$ & 0.013 \\
\hline \multirow{2}{*}{ ENI } & Kaplan-Meier survival & 0.308 & - & 0.005 & 0.005 \\
& Contingency table & 0.165 & - & 0.002 & 0.025 \\
\hline END & Kaplan-Meier survival & 0.099 & 0.005 & - & 0.517 \\
& Contingency table & $<0.001$ & 0.002 & - & 0.327 \\
\hline Observation & Kaplan-Meier survival & 0.052 & 0.005 & 0.517 & - \\
& Contingency table & 0.013 & 0.025 & 0.327 & -
\end{tabular}

END + Adj Tx: Elective neck dissection + adjuvant treatment; ENI: Elective neck irradiation; END: Elective neck dissection.

At our institute, strategy of neck management has been based on the site and $\mathrm{T}$ class of the primary tumor. The decision to treat the neck was made when a risk of subclinical neck disease $\geq 20 \%$ [6]. The types of neck management were in well accord with the risk categories defined by Mendenhall et al. [4] [5]. END + adjuvant treatment was prescribed for patients of the high risk group with unfavorable clinicopathological features: PORT was given to high stage tumor of the oropharynx, hypopharynx and supraglottic larynx, tumor with closed margins; CCRT for tumor of positive margins. Neck treatment either by END or ENI was performed in patients of 
the high risk group with negative tumor margins and those of the intermediate risk group. Close observation was planned for T1 tumor of the oral cavity and glottis. However, ENI was prescribed for T1 tumor of the floor of mouth and T2 glottic tumor. Five-year DSS rates for this series were 61.9\% for END + adjuvant treatment, 85.1\% for END, 44.6\% for ENI and 91.7\% for observation.

The aim of this study was to investigate whether the combined expression of VEGF-A and Ki-67 would provide prognostic information useful for treatment selection or adjustment. Previously, coexpression of VEGF-A and Ki-67 was observed to be an aggressive tumor phenotype for the high likelihood of 6.46 to observe lymph node metastases especially in early stage tumor of the oral cavity, oropharynx and hypopharynx [20]. In this study, coexpression of these markers was an independent prognostic indicator regardless the presence or absence of the nodal diseases. In the setting of N0 neck, marker coexpression was significantly associated with poor patient survival with an HR of $4.97(p=0.013)$. To the best of our knowledge, no other study has been reported for the prognostication of combined marker expression in the context of N0 neck management. However, combined biomarker expression in effective detection of LN metastasis in HNSCC was reported by other. A four-protein signature which was defined by gene products involving tumor growth, invasion and metastasis expressed on primary tumor was used in prediction of LN metastasis and survival of patients with oral SCC [27]. This four-protein signature was defined by epidermal growth factor receptor (EGFR), v-erb-b2 erythroblastic leukemia viral oncogene homolog 2 (HER-2/neu), laminin gamma 2 (LAMC 2) and ras homolog family membrane C (RHOC). The investigators reported that with a combined expression of any 2 or more proteins of the signature could detect LN metastases at sensitivity and specificity of 70\% and $87.5 \%$, respectively and could predict DSS with an adjusted HR of $5.506(p=0.036)$. For bladder cancer, a molecular grading model defined by combined VEGF and Ki-67 expression profiles has been used for predicting tumor recurrence and progression in noninvasive urothelial bladder cancer [28]. The molecular grades were scored: mG3 (both Ki-67 and VEGF were highly expressed), mG2 (either Ki-67 or VEGF was highly expressed) and mG1 (neither Ki-67 nor VEGF was highly expressed). Sensitivity and specificity of the combined markers in predicting tumor recurrence were $91.18 \%$ and $81.25 \%$, respectively as opposed to the lower detection rates obtained by single markers, i.e. $73.53 \%$ for Ki-67 and $66.67 \%$ for VEGF.

In our study, 8 out of 11 patients with VEGF-A and Ki-67 coexpression died regardless the type of neck management. This raised a question whether such an expression profile reflected the microinvasiveness of the primary tumor in addition to the presence of the occult neck diseases. The 5-year DSS rate was $34.9 \%$ for patients with positive nodes in contrasting to the $11.8 \%$ for marker coexpression in the setting of N0 neck. Therefore, marker coexpression in patients with N0 neck might indicate the microinvasiveness of the primary tumor as well as the risk of occult neck disease. Furthermore, among 8 patients who failed, there were only 2 cases receiving the adjuvant treatment of the primary tumor. This led to the postulation that suboptimal treatment of patients with such an aggressive biological feature could be the cause of failure. Henceforth, VEGF-A and Ki-67 coexpression might serve an index in addition to tumor margins in identifying patients who might be benefited from the adjuvant treatment.

On the contrary, there was a question whether neither marker expression would indicate the absence of subclinical neck disease due to a favorable 5-year DSS rate of $81.2 \%$ and therefore would be used as an indicator suggesting neck management by wait-and-observe policy. In this subset of 41 patients, 19 were treated by END (with and without adjuvant treatment), 13 by ENI and 9 by observation. If the subclinical neck disease was absence, one could anticipate the nonsignificant treatment outcomes among these different modalities of neck management. But in fact, the odds of being survived for patients treated by END (with and without adjuvant treatment) were 15 times greater than those treated by ENI. The survival benefit for patients with neither marker expression was therefore due to surgical removal of lymph nodes containing nondividing disseminated tumor cells which were radioresistant.

In the use of END or ENI in treating patients of the same risk category, i.e. intermediate to high risks, a better survival was observed for END with a 5-year DSS rate of $85.1 \%$ as opposed to $44.7 \%$ for ENI. How was this observation compared to other studies? In general, ENI is an option for patients who are poor surgical candidates. Limited studies were conducted to allow the comparison of the effectiveness between END and ENI. In a prospective study using similar clinical criteria, i.e. tumor size and site, in management of cN0 neck for patients with SCC of the oral cavity, the authors reported the 3-year DSS rates of 86\% for END and 67\% for ENI [29]. These survival rates were comparable to those obtained in the current series. Our 3-year DSS rates estimated for END and ENI were $90.4 \%$ and 53.6\%, respectively. Why patient survival from ENI treatment was poorer than 
END. In the ENI treated group, despite the similarity in distributions of tumor site and T class, a few more patients with coexpression of VEGF-A and Ki-67 and the radioresistance of the subclinical neck diseases in patients with tumors expressing neither markers would be factors contributing to the lower rate of tumor control. Although the biomarker study did not provide information for whom should be spared from the neck treatment, it did suggest a treatment adjustment for patients who were originally planned for neck irradiation to consider neck dissection when their tumor expressing neither markers.

In comparison of treatment outcomes between END and observation which involved patients of different risk categories, i.e. intermediate to high risk for END and low risk for observation, survival equivalent between these neck management modalities should not be misjudged as similarity in treatment effects but rather the equivalence in outcomes of neck management in different clinical contexts, i.e. the effectiveness of END in removal of the subclinical neck diseases and the absence of the occult metastasis in close observation. In spite of the favorable treatment efficacy with END, there is still a concern on the unnecessary neck treatment for approximately $70 \%$ of patients who actually do not harbor the metastatic cells in their necks. In indentifying whom to be treated or spared from the neck treatment, we may need a sensitive and reliable method like sentinel node biopsy (SNB) [9]. Nevertheless, the SNB technique, although clinically attractive, is still in the investigational stage for cancer of the head and neck [9] and provides no information regarding to tumor biological behavior.

Head and neck cancers are heterogeneous in anatomical location and biological behavior. We acknowledge our study limitations for the inclusion of cancer arising from the oral cavity and the larynx and the small number of patients. Further study with large sample size is warranted to ascertain the consistency of the findings so as to gain acceptance of the use of these biomarkers as a complementary tool to the standard clinicopathological criteria in selecting the best treatment for individual patients.

\section{Conclusion}

Coexpression of VEGF-A and Ki-67 was a significant prognostic factor independent of modalities of neck management in the setting of N0 neck. Such a pattern of marker expression is a suggestion of an aggressive tumor phenotype implicating the microinvasiveness of the primary tumor in addition to the risk of lymph node metastasis defined previously [20] and may indicate the need of adjuvant treatment for tumor despite its negative margins. On the contrary, neither marker expression was associated with a better outcome of neck treatment by END than by ENI based on the odds of patients being survived 15 times greater and would suggest neck dissection for patient who was originally planned for neck irradiation.

\section{Acknowledgements}

The authors would like to acknowledge the grant support from Mahidol University, Bangkok, Thailand.

\section{References}

[1] Audet, N., Beasley, N.J., MacMillan,C., Jackson, D.G., Gullane, P.J. and Kamel-Reid, S. (2005) Lymphatic Vessel Density, Nodal Metastases, and Prognosis in Patients with Head and Neck Cancer. Archives of Otolaryngology Head and Neck Surgery, 131, 1065-1070. http://dx.doi.org/10.1001/archotol.131.12.1065

[2] Teymoortash, A. and Werner, J.A. (2012) Current Advances in Diagnosis and Surgical Treatment of Lymph Node Metastasis in Head and Neck Cancer. GMS Current Topics in Otorhinolaryngology-Head and Neck Surgery, 11, Article ID: Doc04. http://www.egms.de/en/journals/cto/2012-11/cto000086.shtml

[3] Hosal, A.S., Carrau, R.L., Johnson, J.T. and Myer, E.N. (2000) Selective Neck Dissection in the Management of the Clinically Node-Negative Neck. Laryngoscope, 110, 2037-2040. http://dx.doi.org/10.1097/00005537-200012000-00011

[4] Mendenhall, W.M., Million, R.R. and Cassisi, N.J. (1986) Squamous Cell Carcinoma of the Head and Neck Treated with Radiation Therapy: The Role of Neck Dissection for Clinically Positive Neck Nodes. International Journal of Radiation Oncology Biology Physics, 12, 733-740. http://dx.doi.org/10.1016/0360-3016(86)90030-1

[5] Mendenhall, W.M. and Million, R.R. (1986) Elective Neck Irradiation for Squamous Cell Carcinoma of the Head and Neck: Analysis of the Time-Dose Factors and Causes of Failure. International Journal of Radiation Oncology Biology Physics, 12, 741-746. http://dx.doi.org/10.1016/0360-3016(86)90031-3

[6] Weiss, M.H., Harrison, L.B. and Isaac, R.S. (1994) Use of Decision Analysis in Planning and Management Strategy for the Stage N0 Neck. Archives of Otolaryngology Head and Neck Surgery, 120, 699-702. 
http://dx.doi.org/10.1001/archotol.1994.01880310005001

[7] Jalisi, S. (2005) Management of the Clinically Negative Neck in Early Squamous Cell Carcinoma of the Oral Cavity. Otolaryngologic Clinics of North America, 38, 37-46. http://dx.doi.org/10.1016/j.otc.2004.09.002

[8] Li, X., Shen, Y., Di, B. and Song, Q. (2012) Metastasis of Head and Neck Squamous Cell Carcinoma. In: Li, X., Ed., Squamous Cell Carcinoma, In Tech, Shanghai, 1-31.

http://www.intechopen.com/books/squamous-cell-carcinoma/metastasis-of-head-and-neck-squamous-cell-carcinoma-h $\underline{\text { nscC }}$

[9] Ayman, F.H., Mohamed, E. and Mustafa, G.K. (2013) Management of Clinically Negative Neck in Oral Squamous Cell Carcinoma: A Systemic Review. Journal of Cancer Research and Therapeutic Oncology, 2, 1-12.

http://www.jscholaronline.org/full-text/JCRTO/302/Management-of-the-Clinically-Negative-Neck-in-Oral-SquamousCell-Carcinoma-A-Systematic-Review.php

[10] Roepman, P., de Jager, A., Groot Koerkamp, M.J.A., et al. (2006) Maintenance of Head and Neck Tumor Gene Expression Profiles upon Lymph Node Metastasis. Cancer Research, 66, 11110-11114. http://dx.doi.org/10.1158/0008-5472.CAN-06-3161

[11] Takes, R.P. (2004) Staging of the Neck in Patients with Head and Neck Squamous Cell Cancer: Imaging Techniques and Biomarkers. Oral Oncology, 40, 656-667. http://dx.doi.org/10.1016/j.oraloncology.2003.11.001

[12] Timar, J., Csuka, O., Remenar, E., Repassy, G. and Kasler, M. (2005) Progression of Head and Neck Squamous Cell Cancer. Cancer and Metastasis Reviews, 24, 107-127. http://dx.doi.org/10.1007/s10555-005-5051-5

[13] Chiang, A.C. and Massague, J. (2008) Molecular Basis of Metastasis. New England Journal of Medicine, 359, 28142823. http://dx.doi.org/10.1056/NEJMra0805239

[14] Takes, R.P., Rinaldo, A., Rodrigo, J.P., Devaney, K.O., Fagan, J.J. and Ferlito, A. (2008) Can Biomarkers Play a Role in the Decision about Treatment of the Clinically Negative Neck in Patients with Head and Neck Cancer? Head Neck, 30, 525-538. http://dx.doi.org/10.1002/hed.20759

[15] Lothaire, P., de Azambuja, E., Dequanter, D., et al. (2006) Molecular Markers of Head and Neck Squamous Cell Carcinoma: Promising Signs in Need of Prospective Evaluation. Head Neck, 28, 256-269. http://dx.doi.org/10.1002/hed.20326

[16] Oliveira, L.R. and Ribeiro-Silva, A. (2011) Prognostic Significance of Immunochemical Biomarkers of Oral Squamous Cell Carcinoma. International Journal of Oral Maxillofacial Surgery, 40, 298-307. http://dx.doi.org/10.1016/j.ijom.2010.12.003

[17] Franchi, A., Gallo, O., Boddi, V. and Santucci, M. (1996) Prediction of Occult Neck Metastasis in Laryngeal Carcinoma: Role of Cell Proliferating Nuclear Antigen, MIB-1 and E-Cadherin Immunohistochemical Determination. Clinical Cancer Research, 2, 1801-1808.

[18] Liu, M., Lawson, G., Delos, M., et al. (2003) Prognostic Value of Cell Proliferation Markers, Tumor Suppressor Proteins and Cell Adhesion Molecules in Primary Squamous Cell Carcinoma of the Larynx and Hypopharynx. European Archive Oto-Rhinolaryngology, 260, 28-34.

[19] Myoung, H., Kim, M.J., Lee, J.H., Ok, Y.J., Paeng, J.Y. and Yun, P.Y. (2006) Correlation of Proliferative Markers (Ki-67 and PCNA) with Survival and Lymph Node Metastasis in Oral Squamous Cell Carcinoma: A Clinical and Histopathological Analysis of 113 Patients. International Journal of Oral and Maxillofacial Surgery, 35, 1005-1010. http://dx.doi.org/10.1016/j.ijom.2006.07.016

[20] Boonkitticharoen, V., Kulapaditharom, B., Leopairut, J., et al. (2008) Vascular Endothelial Growth Factor-A and Proliferation Marker in Prediction of Lymph Node Metastasis in Oral and Pharyngeal Squamous Cell Carcinoma. Archives of Otolaryngology Head and Neck Surgery, 134, 1305-1311. http://dx.doi.org/10.1001/archotol.134.12.1305

[21] Mineta, H., Miura, K., Orgino, T., et al. (2000) Prognostic Value of Vascular Endothelial Growth Factor (VEGF) in Head and Neck Squamous Cell Carcinomas. British Journal of Cancer, 83, 775-781. http://dx.doi.org/10.1054/bjoc.2000.1357

[22] O-charoenrat, P., Rhys-Evans, P. and Eccles, S.A. (2001) Expression of Vascular Endothelial Growth Factor Family Members in Head and Neck Squamous Cell Carcinoma Correlated with Lymph Node Metastasis. Cancer, 92, 556-568. http://dx.doi.org/10.1002/1097-0142(20010801)92:3<556::AID-CNCR1355>3.0.CO;2-Q

[23] Cordes, C., Munzel, A.K., Rudolph, P., Hoffmann, M., Leuschner, I. and Gottschlich, S. (2009) Immunohistochemical Staining of Ki-67 Using the Monoclonal Antibody Ki-S11 Is a Prognostic Indicator for Laryngeal Squamous Cell Carcinoma. Anticancer Research, 29, 1459-1466.

[24] Smith, B.D., Smith, G.L., Carter, D., Sasaki, C.T. and Haffty, B.G. (2000) Prognostic Significance of Vascular Endothelial Growth Factor Protein Levels in Oral and Oropharyngeal Squamous Cell Carcinoma. Journal of Clinical Oncology, 18, 2046-2052.

[25] Kyzas, P.A., Stefanou, D., Batistatou, A. and Agnantis, N.J. (2005) Prognostic Significance of VEGF Immunohisto- 
chemical Expression and Tumor Angiogenesis in Head and Neck Squamous Cell Carcinoma. Journal of Cancer Research and Clinical Oncology, 131, 624-630. http://dx.doi.org/10.1007/s00432-005-0003-6

[26] Cerezo, L., Millon, I., Torre, A., Aragon, G. and Otero, J. (1992) Prognostic Factors for Survival and Tumor Control in Cervical Lymph Node Metastases from Head and Neck Cancer. A Multivariate Study of 492 Cases. Cancer, 69, 12241234. http://dx.doi.org/10.1002/cncr.2820690526

[27] Zanarudin, S.N.S., Sach, A., Yang, Y., et al. (2013) Four-Protein Signature Accurately Predicts Lymph Node Metastasis and Survival in Oral Squamous Cell Carcinoma. Human Pathology, 44, 417-426. http://dx.doi.org/10.1016/j.humpath.2012.06.007

[28] Chen, J.X., Deng, N., Chen, X., et al. (2012) A Novel Molecular Grading Model: Combination of Ki-67 and VEGF in Predicting Tumor Recurrence and Progression in Non-invasive Urothelial Bladder Cancer. Asian Pacific Journal of Cancer Prevention, 13, 2229-2234. http://dx.doi.org/10.7314/apjcp.2012.13.5.2229 http://www.koreascience.or.kr/article/ArticleFullRecord.jsp?cn=POCPA9_2012_v13n5_2229

[29] O’Brien, C.J., Traynor, S.J., McNeil, E., McMahon, J.D. and Chaplin, J.M. (2000) The Use of Clinical Criteria Alone in the Management of the Clinically Negative Neck Among Patients with Squamous Cell Carcinoma of the Oral Cavity and Oropharynx. Archives of Otolaryngology Head and Neck Surgery, 126, 360-365.

http://dx.doi.org/10.1001/archotol.126.3.360 